\title{
Lower back pain after recently giving birth: postpartum sacral stress fractures
}

\author{
Luis Saura Beltran • Jenny T. Bencardino
}

Received: 11 July 2010 /Revised: 7 October 2010 /Accepted: 14 October 2010 /Published online: 16 November 2010

(C) ISS 2010

\section{Part II}

Diagnosis

Postpartum sacral stress fractures

\section{Discussion}

Stress fracture is a general term that encompasses both fatigue fractures occurring in normal bone under abnormal or unaccustomed biomechanical load, and insufficiency fractures occurring in weakened bone under normal biomechanical load.

Insufficiency fractures of the sacrum secondary to postmenopausal or age-related osteoporosis are frequent and predicted to triple by the year 2030 secondary to raised awareness, advanced radiological methods of diagnosis, and increasing mean age [1]. In contrast, fractures of the sacrum occurring during pregnancy, labor, or immediately postpartum are rare and only a few case reports have been published in the English-language literature of patients presenting with insufficiency fractures [2], fatigue fractures [3-7], and those where the authors were not sure if they were dealing with fatigue fracture or if there were insufficiency fractures with underlying osteoporosis [8].

One of the first case reports of pregnancy-related sacral insufficiency fracture was attributed to gestational osteoporosis [2]. This patient had decreased bone mineral density

The case presentation can be found at doi:10.1007/s00256-010-1062-6

L. S. Beltran $(\varangle) \cdot$ J. T. Bencardino

Department of Radiology, NYU Hospital for Joint Diseases,

301 East 17th Street,

New York, NY 10003, USA

e-mail: Luis.Beltran@nyumc.org
(BMD) and an increased alkaline phosphatase activity, which was attributed to a physiological phenomenon of pregnancy, vitamin D deficiency, mechanical stress due to the fetus' weight, and a metabolic mechanism induced by long-term heparin therapy ( $>4$ months). Heparin is known to induce osteopenia, which is attributed to a direct effect on osteoclastic activity induced by a dose-dependent calcium homeostasis disorder predisposing to osteoporotic fractures [9].

Pregnancy-related osteoporosis is rare and its pathogenesis is unclear [8]. Patients are predisposed to develop insufficiency fractures in the spine, femoral neck, wrist or clavicle. Decreased serum calcium levels may occur during pregnancy [10] due to decreased levels of 1,25-dihydroxyvitamin $\mathrm{D}_{3}$, decreased calcitonin levels, and the effects of cytokines on bone remodeling.

Subsequent reports of sacral stress fractures related to pregnancy in patients with a normal BMD [5, 8] were published. Risk factors for fatigue sacral fractures during pregnancy and postpartum likely include vaginal delivery of a high birth weight infant, increased lumbar lordosis, excessive weight gain, and rapid vaginal delivery. We were not sure in our case presentation whether we were dealing with a fatigue fracture or an insufficiency fracture because BMD was not measured in our patient. Stress fractures of the sacrum can occur in healthy young individuals other than in relation to pregnancy, particularly in long-distance runners and other athletes.

Imaging findings of pregnancy-related sacral fractures are similar to sacral insufficiency fractures related to involutional osteoporosis with the exception that patients will be women in the reproductive years and have a history of last trimester pregnancy or recent postpartum. Radiographs may be normal or demonstrate unilateral or bilateral areas of sclerosis along the sacral wings (Fig. 1). 
The patient we present also had concomitant osteitis condensans ilii, which was indicated by the presence of triangular-shaped subchondral sclerosis along the iliac side of the sacroiliac joints bilaterally (Fig. 1). MRI typically demonstrates vertical $\mathrm{T} 1$ and $\mathrm{T} 2$ hypointense linear foci along the sacral alae representing fracture lines, and $\mathrm{T} 1$ hypointense and $\mathrm{T} 2$ hyperintense signal in the surrounding bone marrow representing associated edema (Fig. 2). CT will demonstrate increased density along the sacral alae representing the reactive sclerosis along the fracture lines (Fig. 3), and some patients may demonstrate diffuse osteopenia. Dual-energy X-ray absorptiometry (DXA) may be useful in assessing decreased bone mineral density (BMD) T-scores in the lumbar spine and femoral neck [2].

The clinical differential diagnoses include sacroiliitis with inflammatory or infectious causes, osteitis condensans ilii, lumbosacral degenerative spondylosis, and transient regional osteoporosis of the sacrum. In our case, sacroiliitis was a consideration given marked reactive sclerosis on the iliac side of the joints. However, the diagnosis of osteitis condensans ilii was more consistent in our patient due to the presence of only subchondral sclerosis along the iliac side of the sacroiliac joint and absent joint effusion or erosions in conjunction with the clinical history of recent pregnancy and labor. In addition, this patient had sacral fractures demonstrated by the vertical sclerotic lines through the sacral wings away from the articular portion of the sacrum at the sacroiliac joint. MR and CT imaging helped to reliably detect the vertically linear components through the sacral wing away from subchondral bone compatible with sacral fractures and also to better characterize the osteitis condensans ilii component in the posterior iliac bones.

Treatment is usually conservative including bed rest and analgesics until resolution of the pain.

In conclusion, sacral fractures related to pregnancy are uncommon, but should be included in the differential diagnosis of lumbosacral pain during pregnancy or the peripartum period. There are two potential underlying etiologies, which include insufficiency fractures in the setting of osteoporosis of pregnancy or fatigue fractures from unaccustomed stress.

Disclosure The authors declare that they have no conflict of interest.

\section{References}

1. Kannus P, Palvanen M, Niemi S, Parkkari J, Jarvinen M. Epidemiology of osteoporotic pelvic fractures in elderly people in Finland: sharp increase in 1970-1997 and alarming projections for the new millennium. Osteoporos Int. 2000;11(5):443-8.

2. Breuil V, Brocq O, Euller-Ziegler L, Grimaud A. Insufficiency fracture of the sacrum revealing a pregnancy associated osteoporosis. First case report. Ann Rheum Dis. 1997;56(4):278-9.

3. Karatas M, Basaran C, Ozgul E, Tarhan C, Agildere AM. Postpartum sacral stress fracture: an unusual case of low-back and buttock pain. Am J Phys Med Rehabil. 2008;87(5):418-22.

4. Lin JT, Lutz GE. Postpartum sacral fracture presenting as lumbar radiculopathy: a case report. Arch Phys Med Rehabil. 2004;85 (8):1358-61.

5. Rousiere M, Kahan A, Job-Deslandre C. Postpartal sacral fracture without osteoporosis. Joint Bone Spine. 2001;68(1):71-3.

6. Thein R, Burstein G, Shabshin N. Labor-related sacral stress fracture presenting as lower limb radicular pain. Orthopedics. 2009;32(6):447. doi:410.3928/01477447-20090511-01477424.

7. Thienpont E, Simon JP, Fabry G. Sacral stress fracture during pregnancy - a case report. Acta Orthop Scand. 1999;70(5):525-6.

8. Schmid L, Pfirrmann C, Hess T, Schlumpf U. Bilateral fracture of the sacrum associated with pregnancy: a case report. Osteoporos Int. 1999;10(1):91-3.

9. Dahlman TC. Osteoporotic fractures and the recurrence of thromboembolism during pregnancy and the puerperium in 184 women undergoing thromboprophylaxis with heparin. Am J Obstet Gynecol. 1993;168(4):1265-70.

10. Kovacs CS, Fuleihan Gel H. Calcium and bone disorders during pregnancy and lactation. Endocrinol Metab Clin North Am. 2006;35(1):21-51. v. 